\title{
An oncolytic adenoviral vector carrying the tyrosinase promoter for glioma gene therapy
}

\author{
ILYA V. ULASOV ${ }^{1}$, ANGEL A. RIVERA ${ }^{2}$, DIRK M. NETTELBECK ${ }^{3}$, LISA B. RIVERA ${ }^{2}$, \\ J. MICHAEL MATHIS ${ }^{4}$, ADAM M. SONABEND ${ }^{1}$, MATTHEW TYLER ${ }^{1}$, \\ MING WANG ${ }^{2}$, JOANNE T. DOUGLAS ${ }^{2}$ and MACIEJ S. LESNIAK ${ }^{1}$
}

\begin{abstract}
${ }^{1}$ Division of Neurosurgery, The University of Chicago, Chicago, IL; ${ }^{2}$ Division of Human Gene Therapy, Departments of Medicine, Obstetrics and Gynecology, Pathology, and Surgery, University of Alabama at Birmingham, Birmingham, AL, USA; ${ }^{3}$ Department of Dermatology, German Cancer Research Center, Heidelberg, Germany; ${ }^{4}$ Gene Therapy Program, Department of Cellular Biology and Anatomy, Louisiana State University Health Sciences Center, Shreveport, LA, USA
\end{abstract}

Received March 30, 2007; Accepted May 16, 2007

\begin{abstract}
Targeting gene expression to cancer cells remains a challenge for the development of gene and viral therapy for gliomas. Recent studies have highlighted transcriptional targeting as one of the possible solutions to overcome this limitation. In this context, melanoma associated antigens (MAAs) are usually over-expressed in brain tumors in comparison to normal brain tissue. For this reason, we investigated the use of the tyrosinase promoter as a transcriptional element to target oncolytic therapy for gliomas. Tyrosinase mRNA expression was evaluated by qRT-PCR in normal human brain tissue as well as in human glioma specimens. We found that this gene was significantly overexpressed in glioma cell lines and in primary glioma samples. Tyrosinase expression correlated with the grade of the tumor (p-value range: 0.05-0.001). Furthermore, transfection of several cell cultures with human and mouse tyrosinase promoters driving a luciferase reporter gene confirmed the activity of this promoter in mouse and human cells. To evaluate whether tyrosinase-activated conditionally replicative adenoviruses (CRAds) could induce toxicity in glioma cells, two vectors (Ad h/m and Ad24TYR) were tested in a mouse glioma model. C57BL/6 mice underwent intracranial injection of tumor cell line GL261. Survival was used to evaluate efficacy of the tested vectors. Mice receiving $1 \times 10^{9} \mathrm{MOI}$ of Ad $\mathrm{h} / \mathrm{m}$ and Ad24TYR following intracranial tumor implants had a median survival of $46 \pm 3$ days $(\mathrm{p}<0.05)$; in contrast, those treated with medium had a median survival of $31 \pm 2$ days.
\end{abstract}

Correspondence to: Dr Maciej S. Lesniak, Division of Neurosurgery, The University of Chicago, 5841 S. Maryland Ave., MC 3026, Chicago, IL 60637, USA

E-mail:mlesniak@surgery.bsd.uchicago.edu

Key words: glioma, glioblastoma, gene therapy, tyrosinase, promoter, oncolytic virus, adenovirus
These results suggest that injection of tyrosinase CRAds leads to prolongation of survival in mice with experimental brain tumors. The tyrosinase promoter stands as a proof of principle of the potential use of MAA over-expression patterns for targeting novel anti-glioma therapies.

\section{Introduction}

The efficiency of gene and oncolytic therapy for malignant gliomas is presently limited by tumor-specific gene expression. To achieve tumor specificity, one of the approaches consists of exploring the distinct transcriptional profile found in tumor cells. This principle can be applied to target oncolytic adenoviral vectors, so called CRAds (1-4).

Glial and melanocytic cells derive from neuroectoderm (5). Consistent with their common embryological origin, some specific proteins are expressed in both kinds of cells. RT-PCR analysis performed on normal and human brain tumor specimens showed that human gliomas have markedly elevated levels of melanoma associated proteins (MAAs), a group of proteins categorically over-expressed in melanoma cells $(6,7)$. Recently, it has been reported that the mouse glioma cell line, GL261, demonstrates high levels of MAAs as well (8). Tyrosinase, an enzyme that catalyzes the ratelimiting step in melanin synthesis, is an illustrative example of these proteins. Within the CNS, homogenates of substantia nigra have detectable tyrosinase-like activity (9). Tyrosinase promoter activity has been observed not only in pigment cells, but also in embryonic neuroectoderm, migrating neural crest cells, spinal cord cells, and specific cells of the cranial region (10). In addition, the mouse tyrosinase promoter is active throughout murine brain development and has been implicated in neuromelanin formation in the substantia nigra and the developing telencephalon (11). These results demonstrate that tyrosinase promoter activity and tyrosinase expression correlate with regions of rapid cell proliferation in the central nervous system.

In this study, we investigated the level of tyrosinase expression in glioma and non-glioma cell lines as well as primary patient samples. Previous studies have exploited the 
over-expression of tyrosinase to create a CRAd that specifically replicated in melanoma tissue via the incorporation of a tyrosinase enhancer/promoter cassette which drove E1A expression and viral replication $(12,13)$. Here, we describe the application of replication-competent adenoviruses capable of targeting glioma cells under the control of a tyrosinase promoter element. The results obtained in our experiments demonstrate that we can use adenoviral vectors to initiate tyrosinase-activated glioma oncolysis. Furthermore, our findings suggest that using a glioma-specific promoter to drive adenoviral replication is an effective method to prolong the survival of animals with experimental brain tumors.

\section{Materials and methods}

Cells and cell culture. The murine glioma GL261, the human glioma U87MG, melanoma SK-MEL-28, lung carcinoma A549 and embryonic kidney (HEK293) cell lines were obtained from American Type Culture Collection (ATCC, Manassas, VA, USA). The human glioma N.10 cell line was purchased from Japan tissue bank (Tokyo, Japan). Normal human astrocytes (NHA) and fibroblasts were purchased from Clonetics (Cambrex Corporation, NJ, USA). The murine glioma GL261 cell line was a gift from Dr Henry Brem (Johns Hopkins Medical Center, Baltimore, MD, USA). Human primary brain tumor specimens were obtained after resection surgery from patients who were diagnosed with glioma (WHO grade II, and IV). The diagnosis was confirmed by a neuropathologist. The study was approved by the Institutional Review Board at the University of Chicago. All cell lines were maintained in a humidified $37^{\circ} \mathrm{C}$ atmosphere containing $5 \% \mathrm{CO}_{2}$ and cultured with DMEM supplemented with $10 \%$ FBS. The primary cells (NHA and fibroblasts) were maintained according to vendor recommendations. Infections were performed in medium with $2 \%$ fetal bovine serum-FBS (CellGrow, USA).

Plasmid and viruses. A series of plasmids containing the luciferase transgene were generously provided by Dr D. Nettelbeck. The luciferase reporter plasmids were generated based on pGL3 background (Promega, Madison, WI, USA). Luciferase expression was driven under the control of the following transcriptional elements: SV40 promoter (pGL3SV40), mouse tyrosinase (pGL3-mTyr) and human tyrosinase (pGL3-hTyr). The human tyrosinase plasmid construction contained the tyrosinase enhancer element (nucleotides 2014-1811), the promoter element (-209/+51) and the mouse tyrosinase promoters which were inserted into the luciferase reporter vector pGL3-Basic (Promega) to produce the human Tyr and mouse Tyr $(12,14,15)$. The replication-competent vectors: AdWT, Ad24CMV, Ad24TYR, Ad5/3DT, and Ad h/m were described by Nettelbeck et al (13); Ad 24-5/3 by Kanerva et al (16); Ad 5/3 by Tekantv et al (17) and Ad 24 virus was described by Fueyo et al (18). Upon amplification of Ad24CMV, Ad24TYR, Ad5/3DT, and Ad h/m in SK-MEL28 cells, the viruses were purified by routine method with $\mathrm{CsCl}$. The viral titer was determined by measuring OD. Ten-fold dilutions of each virus were titrated on HEK293 cells by the TCID50 method (19). Production of CRAds (AdWT, Ad24, Ad 5/3, Ad24CMV, Ad24TYR, Ad5/3DT, Ad 24-5/3 and
Ad h/m) was $3 \times 10^{10}, 6 \times 10^{10}, 3 \times 10^{9}, 1.85 \times 10^{11}, 7.77 \times 10^{9}$, $8.5 \times 10^{10}, 1 \times 10^{9}, 8.7 \times 10^{10} \mathrm{pfu} / \mathrm{ml}$, respectively.

Real-time quantitative RT-PCR for tyrosinase and midkine expression. To examine human tyrosinase gene expression, human tyrosinase sense and antisense primers were designed from the cDNA sequence of the human tyrosinase gene (Genbank accession no. M27160). We also utilized the midkine gene (Genbank accession no. NM001012333) as a control. The sequences of these primers were: tyrosinase gene: forward primer 5'-GAAATACACTGGAAGGATTTGCTA GTC recognizes exon 2/3; reverse primer 5'-CCAAGGAGC CATGACCAGATCCGA recognizes exon 5. Glyceraldehyde3-phosphate dehydrogenase (GAPDH) was used as a housekeeping gene for internal control. The sequences to amplify the GAPDH gene were: sense 5'-CAACTACATGGTTTACA TGTTCCAA, antisense primer 5'-GCCAGTGGACTCCACG ACGT. The primers recognizing human midkine were: sense 5'-ATGCAGCACCGAGGCTTCCT-3' and antisense: 5'-ATCCAGGCTTGGCGTCTAGT-3' (20). The amplified fragment was detected as a 180-nucleotide amplicon (for tyrosinase) and 418 (for GAPDH and MK); GAPDH was used as reference gene. A 10-fold sterilely diluted pUC19 was used as a standard. Control samples comprised of distilled water or non-reverse-transcribed samples and were negative for both tyrosinase and glyceraldehyde-3-phosphate dehydrogenase transcripts. PCR mixtures were designed to result in a master mix with a final volume of $9 \mu \mathrm{l} /$ reaction containing 1X TaqMan ${ }^{\circledR}$ SYBR green PCR kit (Applied Biosystems), $100 \mathrm{nM}$ forward primer, $100 \mathrm{nM}$ reverse primer, $100 \mathrm{nM}$ probe and $0.025 \% \mathrm{BSA}$. Thermal cycling conditions were as follows: $94^{\circ} \mathrm{C}$ x $2 \mathrm{~min}$ for one cycle, 30 cycles at $92^{\circ} \mathrm{C}$ x $20 \mathrm{sec}, 57^{\circ} \mathrm{C}$ x $30 \mathrm{sec}$, and $72^{\circ} \mathrm{C}$ x $20 \mathrm{sec}$, followed by a plate read at $75^{\circ} \mathrm{C}(21)$. Reactions were carried out in triplicate for each sample using the Opticon DNA Engine thermocycler (M J Research, Waltham, MA). All samples were normalized using the expression level of glyceraldehyde3-phosphate dehydrogenase.

Quantitative analysis of viral replication. GL261 mouse glioma cells $\left(0.5 \times 10^{5}\right.$ cells/well) were grown to $60 \%$ confluence and seeded onto 24-well plates with $1 \mathrm{ml}$ of F-12 Dulbecco's modified Eagle's medium. The next day, the medium was aspirated and the cells were infected with AdWT, Ad24CMV, Ad24TYR, Ad5/3DT, Ad h/m, Ad24-5/3 or Ad24 adenoviral vectors at $10 \mathrm{MOI} / \mathrm{cell}$, and incubated at $37^{\circ} \mathrm{C}$ in a humidified atmosphere for $1 \mathrm{~h}$. The cells were rinsed with PBS and $10 \%$ growth medium was added. For replication analysis, on day 1 , duplicate aliquots of medium and detached cells were subjected to DNA isolation and quantitative PCR. DNA was isolated from cells following standard protocol using DNeasy ${ }^{\circledR}$ Tissue Kit (Qiagen Sciences, MD) and quantitative real-time PCR assay for the E1A gene was performed. The sequences of specific primers used for E1A amplification were: sense 5'-AACCAGTTGCCGTGAGAGTTG-3' and antisense: 5'CTCGTTAAGCAAGTCCTCGATACAT-3'. The PCR was performed with glyceraldehyde-3-phosphate dehydrogenase (GAPDH)-specific primers (TaqMan GAPDH control reagent; Applied Biosystems) to create an internal standard. Quantification using SYBR Green PCR Master Mix (Applied Bio- 
systems, CA, USA) was performed according to vendor recommendations. Data are reported as ratio of E1A copy number per GAPDH copy.

Transient transfection assay. The human glioma and nonglioma cell lines were seeded on 6-well plates and the next day transfected with Lipofectamine 2000 reagent (Invitrogen). Each cell line was transfected with 4 different plasmids: pGL3, pGL3-SV40, pGL3-hTyr, and pGL3-mTyr in triplicates. The basic pGL3 plasmid and pEGFP plasmid were used as negative and positive controls, respectively, for transfection analysis. The DNA used for transfection was $5 \mu \mathrm{g} /$ well. After 4-h incubation, cells were rinsed with PBS and 10\% fresh growth medium was added to each well. Forty-eight hours later, expression of luciferase genes was determined by Luciferase reporter assay (Promega, WI). Data are presented as fold of SV40 activity after normalization to total protein.

Tissue slice culture and viral infection. The Krumdieck tissue slicing system (Alabama Research \& Development) was used following both the manufacturer's instructions and previously published techniques (22). Brain tissue slices were placed into 6-well plates (1 slice per well) containing $2 \mathrm{ml}$ of $10 \%$ complete culture media (DMEM). The plates were then incubated at $37^{\circ} \mathrm{C} / 5 \% \mathrm{CO}_{2}$ in a humidified environment for up to $48 \mathrm{~h}$. A plate rocker set at $60 \mathrm{rpm}$ was used to agitate slices and ensure adequate oxygenation and viability.

All viral infections were done at 500 MOI in media supplemented with FCS. Cell number for tissue slices was estimated at $1 \times 10^{6}$ cells/slice based upon a 10-cell thick slice $(\sim 250 \mu \mathrm{m})$ and 8-mm slice diameter. Infections were allowed to proceed overnight and on subsequent day the medium was removed and replaced with complete culture media. Slices were removed 24 or $48 \mathrm{~h}$ post-infection and tissue was subjected to quantitative PCR analysis.

In vitro cytopathic effect. SK-MEL-28, human fibroblast, normal human astrocytes, and GL261 cells $\left(5 \times 10^{4}\right)$ were seeded onto 24-well plates and were either mock infected or infected with different amounts (1000, 100, 10 and 1 MOI per cells) or recombinant viruses in $0.5 \mathrm{ml}$ DMEM containing $2 \%$ fetal calf serum. After $1 \mathrm{~h}$, the media was removed and the cells were maintained in growth media. When cell lysis was observed for Ad24 or Ad24-5/3 viruses at MOI 1 (14 days post infection), the cells were fixed and stained with $1 \%$ crystal violet in $70 \%$ ethanol for $45 \mathrm{~min}$, followed by washing with tap water to remove excess color.

Anti-tumor activity in vivo. The anti-tumor effect of our replication-competent viruses was studied in C57BL/6 mice. Female mice ( $n=10 /$ group) at 5-6 weeks of age underwent intracranial inoculations with $1 \times 10^{6}$ GL261 cells suspended in 5 microliters of RPMI media (23). Briefly, mice were anesthetized with an intraperitoneal injection of $0.1 \mathrm{ml}$ of a stock solution containing ketamine hydrochloride $25 \mathrm{mg} / \mathrm{ml}$, xylazine $2.5 \mathrm{mg} / \mathrm{ml}$, and $14.25 \%$ ethyl alcohol diluted 1:3 in $0.9 \% \mathrm{NaCl}$. For stereotactic intracranial injections of tumor cells, the surgical site was shaved and prepared with $70 \%$ ethyl alcohol and Prepodyne solution. After a midline incision, a 1-mm right parietal burr hole centered $2 \mathrm{~mm}$ posterior to the coronal suture and $2 \mathrm{~mm}$ lateral to the sagittal suture was made. Animals were then placed in a stereotactic frame and $1 \times 10^{5}$ GL261 tumor cells were delivered by a 26-gauge needle to a depth of $3 \mathrm{~mm}$ over a period of $3 \mathrm{~min}$. The total volume of injected cells was $5 \mu$. The needle was removed, the site was irrigated with sterile $0.9 \% \mathrm{NaCl}$, and the skin was sutured with 4.0 nylon. Once the tumor was established (5 days post implantation), mice were randomized into 6 groups. A total of $1 \times 10^{9} \mathrm{MOI}$ were administered to the experimental groups by an intratumoral injection. Control mice received saline injections. At day seven, 3 mice per group were sacrificed, plasma was separated from the blood immediately after collection and subjected to measurement of aspirate amino-transferase (AST), alanine amino-transferase (ALT) and platelets (PLT).

Statistical analyses. Data are presented as mean values \pm standard deviation. Statistical differences among groups were assessed with a two-tailed Student's t-test. $\mathrm{p}<0.05$ was considered significant. The anti-tumor effect of each CRAd was measured based with a Kaplan-Meier survival curve and statistical significance was determined by the Kruskal-Wallis nonparametric analysis of variance followed by the nonparametric analog of the Newman-Keuls multiple comparison test.

\section{Results}

Analysis of tyrosinase mRNA in glioma. We examined tyrosinase mRNA expression in glioma and normal tissues by quantitative RT-PCR, using the expression of the midkine (MK) gene as an internal positive control because of its characteristic over-expression in gliomas $(2,24,25)$. As shown in Fig. 1, the levels of tyrosinase activity in U87MG and A172 were highest among all brain tumor cell lines tested $(\mathrm{p}<0.05)$. Expression levels varied from $9.6 \times 10^{3}$ (Kings) and $6.2 \times 10^{4}$ (U373MG) to $2.44 \times 10^{6}(\mathrm{U} 87 \mathrm{MG})$ and $6.6 \times 10^{5}$ (A172) copies/ng RNA. Tyrosinase levels detected in primary tissue samples were 5- to 11-fold lower than levels detected in SK-MEL-28 cells $(\mathrm{p}<0.05)$. In all glioma cell lines, the MK gene expression profiles were significantly higher than those of tyrosinase (100-1000-fold). Low-grade tissue samples showed minimal tyrosinase and MK activity whereas high grade GBM samples showed significantly higher levels of tyrosinase and MK expression.

Given the higher expression of tyrosinase mRNA among high grade tumors, we hypothesized that tyrosinase expression may vary with the grade of tumor. To test this hypothesis, we used the mRNA expression of MK and tyrosinase to calculate an MK:tyrosinase ratio to correlate the level of tyrosinase expression with tumor malignancy. Passaged glioma cells contain high ratios of $\mathrm{MK}$ to tyrosinase mRNAs: from 354.6 (U87MG) to $4.3 \times 10^{6}$ (Kings) while primary tumors demonstrated low amount of both targets: 0.14 (astrocytoma grade 2) to 2.2 (GBM grade 4) Table I.

Functional analysis of tyrosinase promoter activity in glioma. Since we were able to demonstrate that glioma cells express significant amount of tyrosinase mRNA, we postulated that the promoter elements responsible for driving tyrosinase 


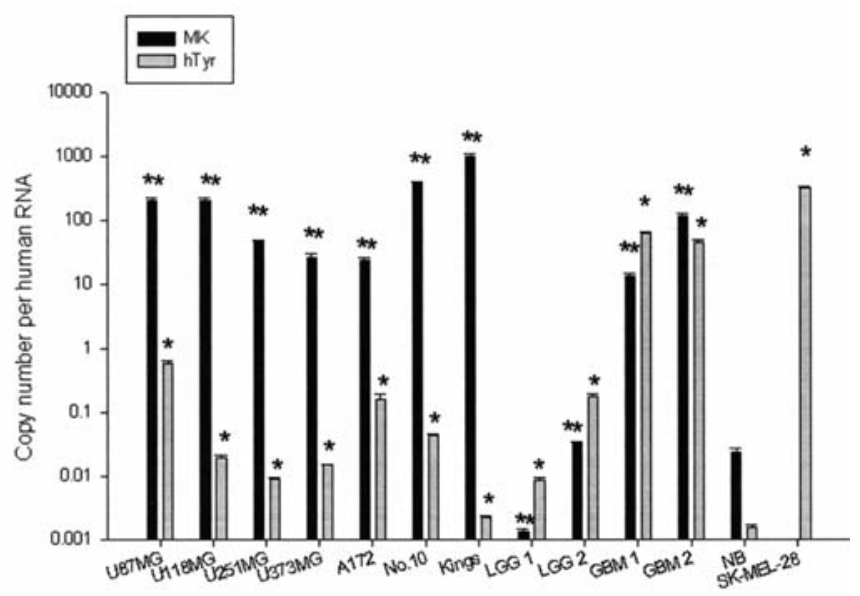

Figure 1. Cell specificity of tyrosinase mRNA expression. RNA was isolated from the cell lines and patient specimens and real-time PCR was performed to determine the quantitative levels of human tyrosinase (hTyr) and MK mRNAs. Two separate experiments were performed for detection of mRNA copy number normalized to the level of GAPDH. Glyceraldehyde-3phosphate dehydrogenase was selected as an endogenous RNA control to normalize for differences in the amounts of total RNA. NB-normal brain specimen; LGG 1 and LGG 2 (WHO grade II), GBM 1 and GBM 2 (WHO grade IV) are primary brain tumor specimens obtained from patients. SK-MEL-28 melanoma cell line expresses high level of human tyrosinase (positive control). Gene expression for hTyr (solid bars) and MK (open bars) are shown as mean $\pm \mathrm{SD}$. The ${ }^{(*,}$ and ${ }^{* *}$, indicate a $\mathrm{p}<0.05$ for tyrosinase and $\mathrm{MK}$ in comparison with NB expression.

Table I. Gene expression profiles of MK:tyrosinase detected in the cells.

\begin{tabular}{ccc}
\hline No. & Cells & Ratio MK:TYR \\
\hline 1 & U87MG & $355 \pm 36.21$ \\
2 & U118MG & $10350 \pm 781$ \\
3 & U251MG & $5078.8 \pm 530.6$ \\
4 & U373MG & $1766 \pm 141.4$ \\
5 & A172 & $147.1 \pm 5.91$ \\
6 & No.10 & $8377.7 \pm 925.1$ \\
7 & Kings & $43652 \pm 728.1$ \\
8 & LGG 1 & $0.154 \pm 0.14$ \\
9 & LGG 2 & $0.181 \pm 0.016$ \\
10 & GBM 1 & $0.222 \pm 0.06$ \\
11 & GBM 2 & $2.52 \pm 0.39$ \\
12 & NB & $0.154 \pm 0.17$ \\
\hline
\end{tabular}

transcription/expression may exhibit equal or more transcriptional activity than the tyrosinase gene itself. To investigate the ability of the tyrosinase promoter to stimulate adenoviral transcription and protein expression in glioma cell lines, we transiently transfected glioma and non-glioma cell lines (HEK293, NHA) with plasmids containing a tyrosinase promoter/enhancer expression cassette driving luciferase expression. We chose the SK-MEL-28 and B16 cell lines as positive controls since tyrosinase expression has been

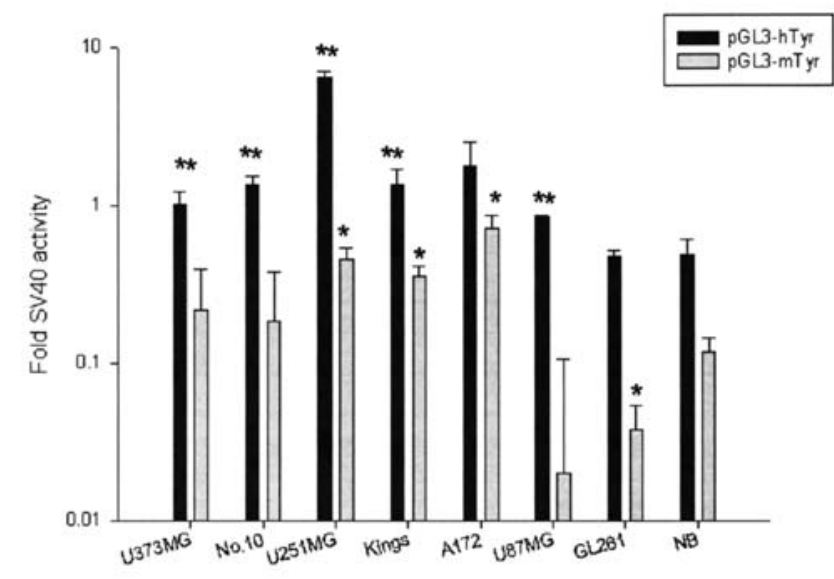

B

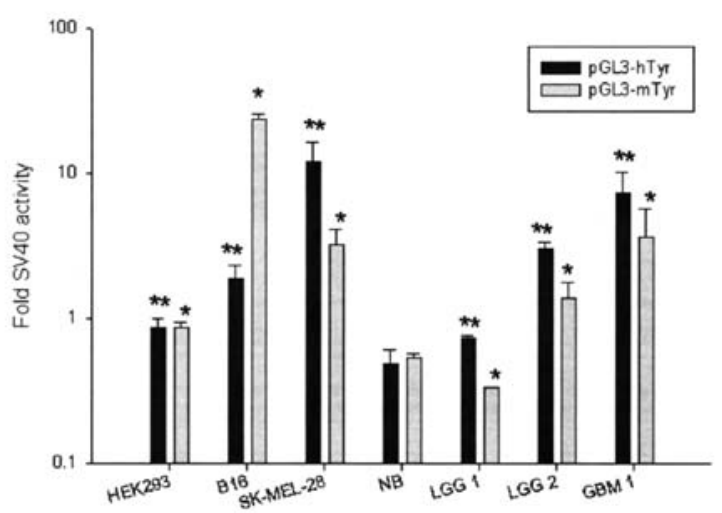

Figure 2. Characterization of tyrosinase promoter activity in gliomas and non-glioma cells. Luciferase activity in 7 human gliomas (A), primary gliomas and non-glioma cells (B) after transient transfection. All cancer cell lines transfected with the GL3-human (pGL3-hTyr) and GL3-mouse promoter (pGL3-mTyr) constructions were incubated for $48 \mathrm{~h}$. The level of luciferase activity is shown relative to the luciferase activity in cells transfected with the GL3 basic plasmid. All cell lines were transfected with pGL3-SV40. The results represent the mean of three experiments of fold SV40 activity. All means were calculated after normalization to proteins. The ${ }^{* *}$, and ${ }^{* * *}$, indicate a $\mathrm{p}<0.05$ for tyrosinase and MK in comparison with NB expression.

demonstrated in both cell lines $(26,27)$. To depict the relative transcriptional activity mediated by the tyrosinase promoters/ enhancers, we normalized promoter-mediated transcriptional activity to the transcriptional activity of the SV40 promoter. As shown in Fig. 2A, seven different glioma cell lines (U373MG, No. 10, Kings, A172, U87MG, and GL261) had similar activities for the human tyrosinase (1.36-1.77-fold over SV40, respectively) promoter and lower activities for the mouse tyrosinase promoter. Human Tyr promoter exhibited the highest activity (6.5-fold over SV40 activity) in U251MG, but this level was still lower than levels detected in SK-MEL-28 cells (Fig. 2B). Surprisingly, GL261 exhibited high levels of human tyrosinase promoter activity vs. mouse tyrosinase. The luciferase expression exhibited by the mouse tyrosinase plasmid was significantly lower than the expression detected in B16 cells and significantly lower than SV40 activity. 
Table II. Characteristics of CRAds.

\begin{tabular}{|c|c|c|c|c|c|c|}
\hline No. & Vector & Vector replication & E1A region/promoter & E4 region/promoter & Fiber & Ref. \\
\hline 1 & AdWT & Competent & wt & wt & wt & $(13)$ \\
\hline 2 & Ad5/3 & Competent & wt & wt & Shaft5-knob3 & (17) \\
\hline 3 & $\operatorname{Ad} 24$ & Competent & $\Delta \mathrm{pRB}$ & wt & wt & $(18)$ \\
\hline 4 & $\operatorname{Ad} 24-5 / 3$ & Competent & $\Delta \mathrm{pRB}$ & wt & Shaft5-knob3 & (16) \\
\hline 5 & Ad24CMV & Competent & $\Delta \mathrm{pRB} /$ human CMV & wt & wt & (12) \\
\hline 6 & Ad24TYR & Competent & $\Delta \mathrm{pRB}$ t/human Tyr+enhancer & wt & wt & (12) \\
\hline 7 & Ad5/3DT & Competent & $\Delta \mathrm{pRB}$ t/human Tyr+enhancer & Mouse Tyr+enhancer & Shaft5-knob3 & (12) \\
\hline 8 & $\mathrm{Ad} h / \mathrm{m}$ & Competent & $\Delta \mathrm{pRB} \mathrm{t} /$ human Tyr+enhancer & Mouse Tyr+enhancer & wt & $(12)$ \\
\hline
\end{tabular}

Next, we examined the promoter activity in non-glioma cells and primary glioma tumors. pGL3-hTyr showed a higher activity in the melanoma cell line SK-MEL-28 (12-fold over SV40 activity) and B16 (1.86-fold). As seen in Fig. 2B, in B16 mouse melanoma, the human tyrosinase promoter displayed a relatively higher activity than in non-melanoma cell lines (293, NHA). The activity was reduced compared with the SV40 promoter ( 0.48 and 0.86 times relatively). In human primary tumor cells, the human promoter exhibited increased level of expression compared to pGL3-mTyr. In GBM sample, we observed significant expression of luciferase induced by human tyrosinase promoter vs. mouse tyrosinase (15.1 \pm 2.61 and $6.64 \pm 0.21$-fold).

Cytolytic effect of virus replication in glioma cells. Previously, we demonstrated how CRAd utilizes the transcriptional activity of the tyrosinase promoter in SK-MEL-28 cells to specifically inhibit melanoma cell proliferation (13-15). Having characterized the high transcriptional activities of tyrosinase and its promoters in glioma cells, we thought it was feasible the same CRAd would be effective in inhibiting glioma cell proliferation. Thus, we evaluated the ability of several recombinant CRAds to inhibit murine GL261 and human U87MG glioma proliferation. SK-MEL-28 was used as a positive control for viral cytolytic activity. Gliomas and human melanoma cell lines were treated with a panel of adenovirus vectors at MOI of 1000, 100, 10, 1 per cell, using AdWT and Ad24CMV as control viruses (Table II). Cytotoxicity was detected by crystal violet assay, which reflects the relative cytolytic capacities of each adenovirus. As shown in Fig. 3, SK-MEL-28 cell line was permissive to the viral infection of Ad24, Ad24CMV, Ad5/3, Ad5/3DT, Ad24TYR and non-permissive to Ad24-5/3 competent vector. Ad24 demonstrated the highest oncolytic potential among all tested vectors. Fourteen days post infection, Ad24TYR induced a CPE more efficiently than other tyrosinasecontrolled CRAd vectors such as Ad h/m and Ad5/3DT, indicated by the disrupted monolayer at MOI of 1 . This activity was similar to the cytolytic activities exhibited by Ad24 and Ad5/3.

We also infected the human U87MG, and murine GL261 glioma cell lines with CRAds at the same doses used in the
A

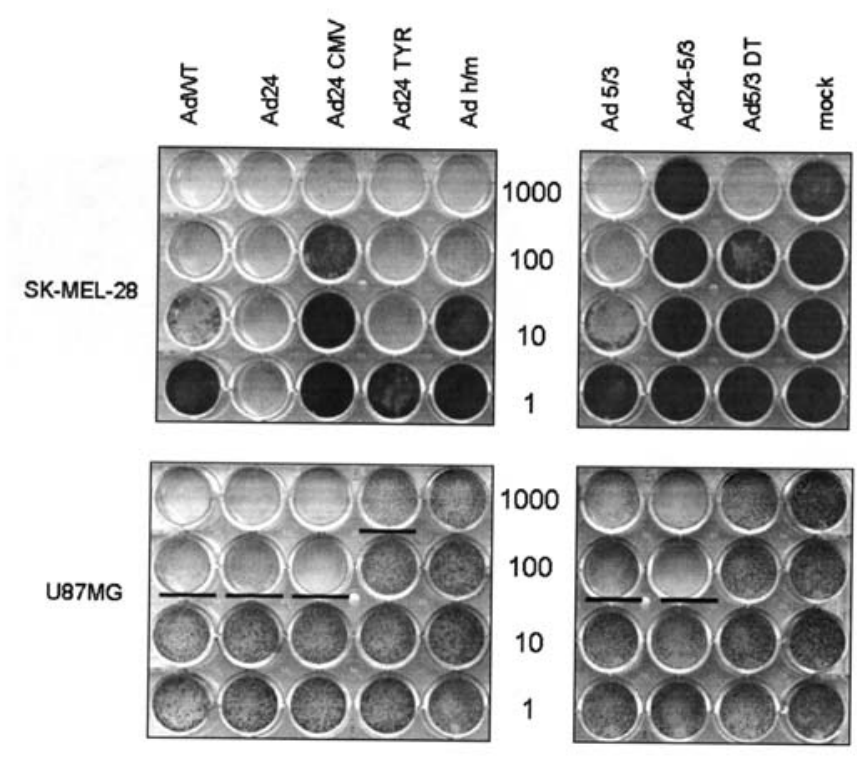

B

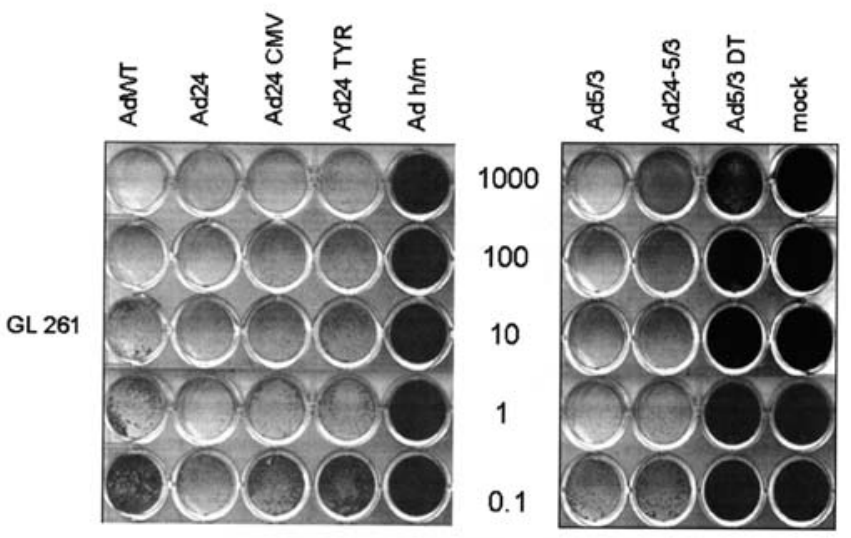

Figure 3. Sensitivity of glioma and non-glioma cells to the CRAd infection. The human melanoma SK-MEL-28 (A) and mouse glioma GL261 (B) cells were treated with CRAds at indicated titer (MOI): 100, 10, 1 and 0.1. Fourteen days later cells were fixed and stained with $2 \%$ crystal violet in $70 \%$ ethanol to visualize killing effect. All experiments were performed twice independently and the data represent one of the experiments. 
A

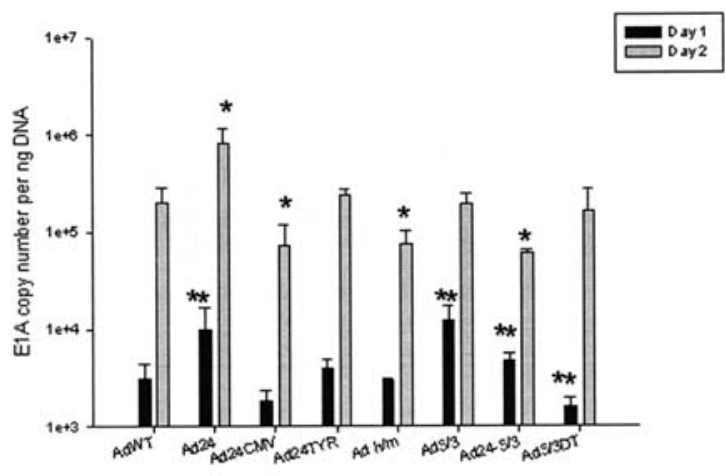

B

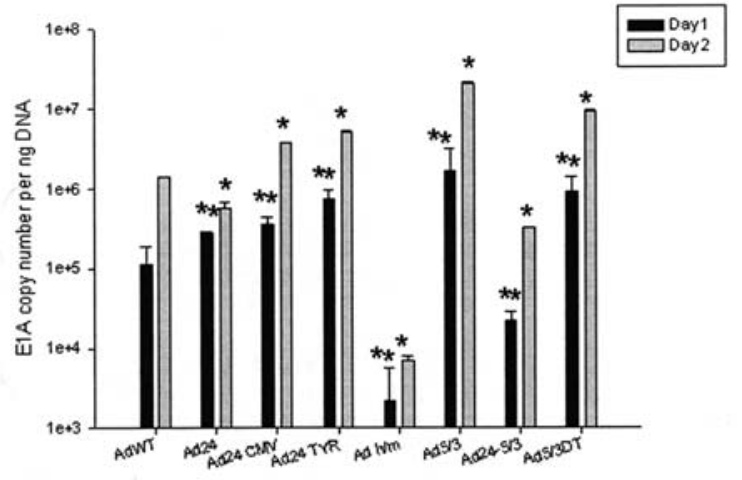

Figure 4. Selective replication of adenoviral vectors in mouse GL261 (A) and primary human glioma specimen (B) cells. (A) GL261 cells were plated the day before infection. Next day, they were infected with the CRAds. After $1 \mathrm{~h}$, infection media was replaced with growth media. DNA was isolated from adherent infected cells on days 1 and 2 after infection. Total DNA was subjected to real-time PCR to determine E1A copy numbers. The asterisk indicates a $\mathrm{p}<0.05$ for the comparison of E1A copies expressed by both CRAds to the level of expression detected in AdWT-infected cells. (B) Primary glioma specimen were prepared and infected as described in Materials and methods. Total DNA was isolated at days 1 and 2 postinfection and E1 copy number was quantified.

previous experiment. In this experiment, U87MG was sensitive to infection with AdWT, Ad24, Ad24CMV, Ad5/3, and Ad24-5/3. Thus, for these vectors we observed significant killing effect at 100 MOI. Nevertheless, Ad24TYR demonstrated 10 times less activity than AdWT. The other interesting finding was observed in GL261 experiment. Ad24 vector exhibited the strongest killing effect among all tested vectors at low dilution. Similar to Ad24 killing effect, Ad24CMV, Ad5/3, Ad24-5/3, Ad24TYR exhibited cytotoxicity at lower dilution-1 MOI, whereas Ad h/m and Ad5/3DT were not effective even at high dose - 1000 MOI.

Ad24TYR shows tumor specific replication in GL261 and primary human specimens. Once we had established the oncolytic capacities of our CRAds by their CPEs, we then wished to evaluate the cytolytic specificity of the tyrosinase recombinant CRAds and determine if the killing effect of these CRAds was in fact due to tyrosinase promoter/enhancermediated viral replication. Thus, we infected mouse glioma

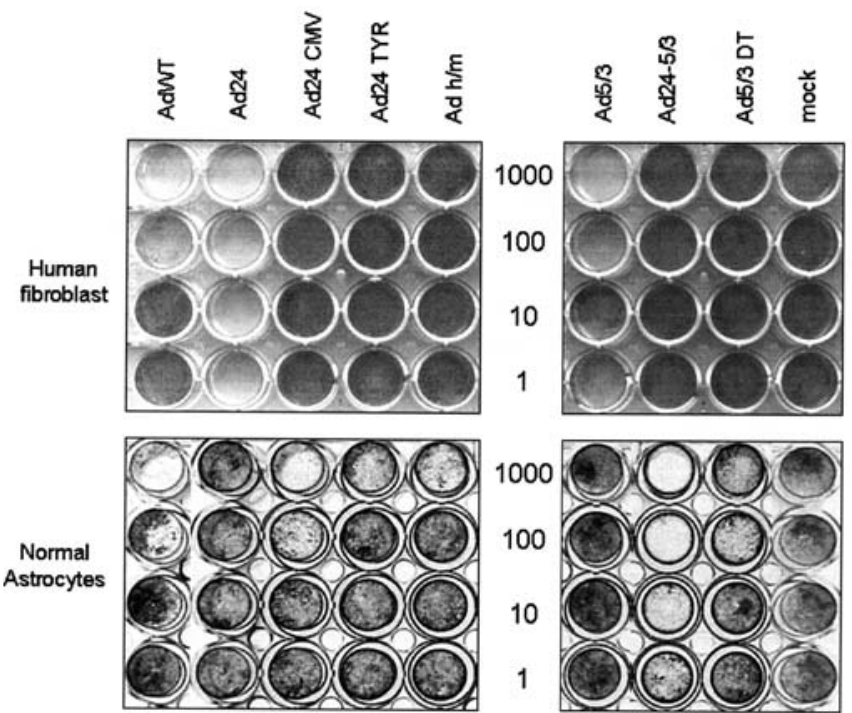

Figure 5. Cytopathic effect induced after infection with CRAds. Normal human fibroblasts (HF) and astrocytes (NHA) were plated the day before infection. Cells were infected the next day with CRAd in different concentrations as indicated and stained with crystal violet after incubation for 14 days.

GL261 cells with our panel of CRAds and calculated the relative number of E1A transcripts 24 and $48 \mathrm{~h}$ post-infection. As seen in Fig. 4A, Ad24 and Ad5/3 showed the greatest level of E1A copy number expression compared with AdWT, whereas, Ad24CMV and 5/3DT showed the lowest level of replication at $24 \mathrm{~h}$. At $48 \mathrm{~h}$ post-infection, the level of E1A expression patterns were similar to those at $24 \mathrm{~h}$. Among tyrosinase-controlled vectors, Ad24TYR exhibited highest potential for replication in GL261 cells.

To ascertain whether these CRAds have any potential for application in the clinical arena, we infected primary glioma samples resected from patients diagnosed with grade IV glioma. We infected 5 micron sections and measured the level of E1A expression 24 and $48 \mathrm{~h}$ post-infection as in the previous experiment. As presented in Fig. 4B, Ad h/m and Ad24-5/3 yielded lower E1A transcripts at both time periods. The other vectors revealed increased levels of E1A expression at 24 and $48 \mathrm{~h}$. Ad5/3, Ad5/3DT and Ad24TYR exhibited the highest replication activity when compared to other vectors including Ad24CMV and Ad24. The data suggest that the tyrosinase promoter induces viral replication in a cell-specific manner and support our data obtained in our quantitative RT-PCR analyses.

Ad24TYR demonstrated low activity in human non-glioma primary cells. We examined the potential toxic effect of our CRAds in normal brain samples to further determine the transcriptional targeting capabilities of the recombinants. We did this by conducting infections in cultures of passaged normal fibroblasts and human astrocytes (NHAs). In fibroblasts, crystal violet staining 14 days after infection revealed that the cytotoxicity of Ad5/3 and AdWT was more than 100-fold less when compared to the cytotoxicity rendered by $\mathrm{Ad} 24$ vector (Fig. 5). NHA cells were vulnerable to infection by Ad24-5/3, AdWT and Ad24CMV viruses. Among the tyrosinase-modified CRAds, a significant killing effect was observed in astrocytes infected with the Ad5/3DT vector 
$\mathbf{A}$

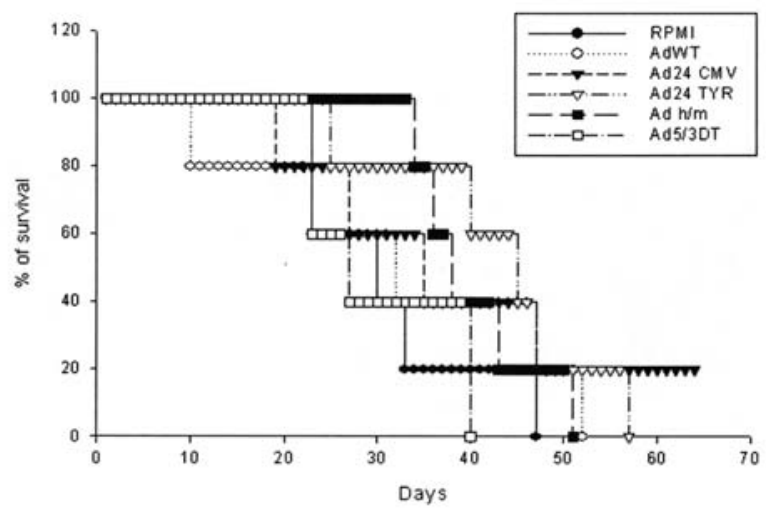

B

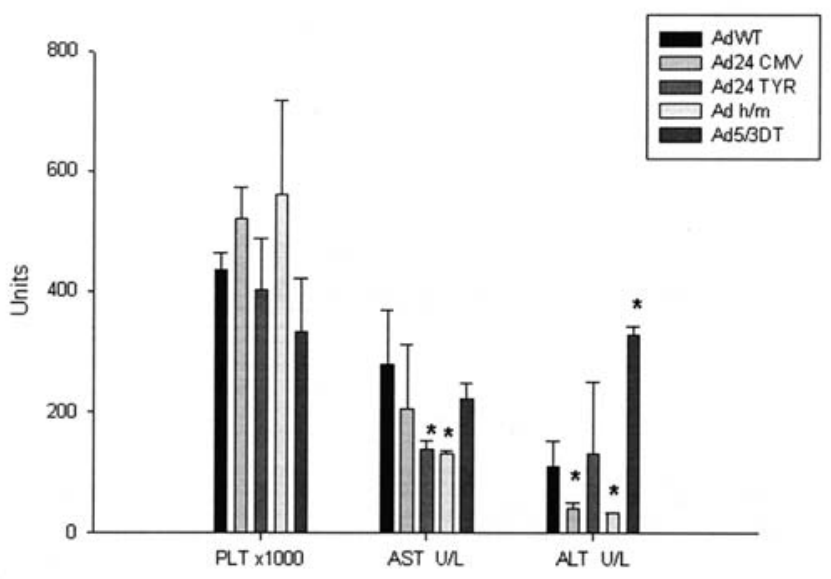

Figure 6. The therapeutic effect of tyrosinase CRAds in C57BL/6 mice with established GL261 xenografts. To establish intracranial tumors, 10 mice received $1 \times 10^{6}$ of GL261 mouse glioma cells in $5 \mu 1$ of RPMI media. Five days later, the mice were divided into 6 groups and treated with the recombinant vector $\left(10^{9} \mathrm{MOI} /\right.$ mouse $)$ or wild-type adenovirus. (A) Kaplan-Mayer survival curve of i.c. injected mice. (B) Evaluation of toxicological characteristics after 7 days after CRAds injections. ALT, AST and PLT were determined for each mouse and presented as group's average \pm SD .

whereas Ad24TYR and Ad h/m showed the lowest toxicity in the context of NHA. At the same time, Ad24CMV, Ad24TYR, Ad h/m, Ad24-5/3 and Ad5/3DT showed little cytotoxicity in primary cultures, suggesting that these CRAds exhibit cell-specific replication patterns.

Ad24TYR induces prolonged survival in mice with experimental brain tumors. Finally, we examined the anti-glioma effect of our CRAds in an intracranial murine glioma model. Based on the crystal violet cytotoxic assays conducted in NHA and GL261 cells, we selected the vectors that demonstrated the lowest ability to infect and kill normal tissue, such as fibroblasts and NHA, but at the same time exhibited the highest therapeutic index in vitro mediated by the capability for specific replication in glioma tissue. After determining minimal toxic doses, we treated GL261 established xenografts with our selected CRAds. As shown in Fig. 6A, the treatment with Ad24TYR and Ad h/m improved the median survival of the mice over RPMI-treated group
(39 and 46 days, respectively $\mathrm{p}<0.05$ ) whereas Ad24CMV and Ad5/ 3DT did not show significant anti-tumor effects compared with the control group (27 and 36 days, respectively $\mathrm{p}>0.05)$. No significant difference in survival was observed when we compared the RPMI- and AdWT-treated group (31 vs. 33 days). To evaluate the relative hepatotoxicities of Ad24CMV, Ad24TYR, AdWT, Ad h/m, and Ad5/3DT, we assessed the presence of liver enzyme abnormalities 7 days after infection. As presented in Fig. 6B, we found no significant hematological abnormalities in any group. However, we detected a slightly increased amount of platelets (PLT) (in mice receiving $\mathrm{Ad} 24 \mathrm{CMV}$ and $\mathrm{Ad} \mathrm{h} / \mathrm{m}$ injections). Of note, the level of AST was within the normal range and lower than after AdWT exposure. The injection of Ad5/3DT led to increased amount of ALT expression ( 2.5 -fold over AdWT group data).

\section{Discussion}

Despite the progress of glioma treatment in recent years, the median survival of patients remains less than two years (28-30). Therefore, great need exists for designing new therapeutic techniques and modifying old ones. Gene therapy that consists of using viral vectors for the treatment of glioma is a promising strategy. Currently, the most commonly investigated variety of viral vectors designed to transduce and selectively replicate in malignant glioma are herpes viruses $(31,32)$, adenoviral vectors $(3,33)$, retroviruses (34-36) reoviruses (37), and alphaviruses (38). Conditionally replicative adenoviral vectors are the most commonly tested vehicles for gene therapy. Their potential to effectively replicate and spread among tumor cells makes them an attractive tool to induce tumor toxicity.

Tyrosinase is a member of the melanoma-associated antigen (MAA) family and is generally found to be produced by melanocytes $(6,39-41)$. It has been shown that tyrosinase related proteins are expressed in $51 \%$ of glioma specimens (42). In our study, we used the mRNA expression of tyrosinase promoters to assess the capability of such promoters to achieve transcriptional targeting by conditionally replicating adenoviruses. Tyrosinase deserves some attention because melanocytes and glial cells both display little permissiveness for adenovirus replication but express common proteins (neuromelanin) during their embryological development $(43,44)$. Therefore, given the shared proteomic profiles between melanocytes and glial cells during their highly proliferative embryological development, logical extrapolations can be made concerning the similar expression profiles of the two cell types during tumorigenesis. That is, if tyrosinase expression is found to be prevalent during the embryological differentiation of the two cell types, then, tyrosinase may also be similarly over-expressed during melanoma and glioma proliferation.

We proposed that the human tyrosinase promoter expression levels should be consistent with the tyrosinase mRNA expressions in cell lines as well as patient specimens. Our in vitro studies showed that human tyrosinase is active in all tested human glioma cells. Moreover, primary glioma samples received from patients demonstrated high levels of tyrosinase activity. Reverse transcription-PCR 
assays revealed that in normal brain specimens the level of tyrosinase mRNA is low; whereas in passaged glioma cells and patient samples, the amounts of tyrosinase mRNA increases from 100 up to $10^{4}$ copies/ng RNA. To assess the relative amount of tyrosinase elements expressed, we chose to compare MK mRNA expression to tyrosinase element expression because MK exhibits high levels of expression in all glioma samples as well as many other cancers. In vitro quantification analysis shows about 100-1000-fold increased level of MK. Of note, significant mRNA levels were detected in primary samples. These results provide a more realistic representation of the tyrosinase expression patterns of malignant glioma. Thus, the level of MK is still the highest, but the level of tyrosinase is comparable and about 2-3 times lower than the levels detected in our positive control sample - SK-MEL-28 (human melanoma cell line).

These initial results can be analyzed to represent the activity of tyrosinase promoters. But to explore this finding and verify that tyrosinase promoter activity correlates with high levels of tyrosinase expression in glioma, we conducted a reporter transfection assay. Our results with the human and mouse tyrosinase promoters indicated activities that correlated to the tyrosinase transcription activities assayed in the RT-PCR tests. The activity of human tyrosinase promoters is especially comparable to the SV40 promoter activity in U251MG cells. The data obtained in primary human glioma samples further corroborate our results which showed increased tyrosinase promoter activity and human tyrosinase RNA expression in U87MG, A172 and U251MG cells.

To obtain tissue-specific replication by competent vectors we constructed several adenoviral vectors containing tyrosinase (human, mouse or both) promoters which drive E1A expression in a tissue specific manner. We hypothesized that employing tyrosinase promoters for treating brain tumors would enhance the localization of viral replication to neoplasms compared with AdWT. In this study, we evaluated the efficacy of Ad24TYR, Ad5/3DT and Ad h/m in vitro in several glioma cell lines, in normal human fetal astrocytes (NHA), fibroblasts (HF) and GL261 murine glioma model. Our results indicated that human U87MG and mouse GL261 glioma cells are very sensitive to Ad24TYR at 1000 and $1 \mathrm{MOI} /$ cell infection. To detect viral toxicity mediated by tyrosinase CRAds, we performed infection of NHA and fibroblasts with adenoviral vectors. As shown in this experiment, the control vector Ad24 induced toxicity even at low dose (in human fibroblast) but was non-toxic to NHA, whereas AdWT infected both type of cells with equal efficiency. Among tyrosinasecontrolled CRAds, we have shown that Ad5/3DT and Ad $\mathrm{h} / \mathrm{m}$ demonstrated similar toxicities only in NHA, similar to AdWT. Ad24CMV induced more toxicity than Ad24, which suggests a lack of oncolytic specificity of the CMV promoter in this infection. Surprisingly, the toxicity of Ad24TYR was 10 times less than Ad24CMV. This finding suggests the employment of tyrosinase promoters in CRAd replication renders a higher therapeutic index than the use of CMV CRAds.

Despite the putative restricted manner of Ad24CMV replication, we validated that activity in normal tissue. The results obtained confirm those obtained by us and StoffKhalili et al (45) and support conclusions that AdWT and
Ad24CMV induce toxicity to NHA. To evaluate the varying levels of selective replication, we compared the activities of tyrosinase-controlled CRAds by infection of fibroblasts and NHA, two different cell types detected in brain tissue. According to Fueyo et al (46), NHA are not permissible to viral replication by Ad24RGD. This result gives further depth to our study by demonstrating the augmented capacity for selective replication of the Ad24TYR vector. In our study, we observed significant toxicity mediated by Ad24$5 / 3$ vector. In contrast, Ad24TYR demonstrated the lowest toxicity in NHA and none in fibroblasts, which correlates with activity of human tyrosinase promoter in normal brain tissue. This finding also supports the idea that incorporation of a CMV promoter into E1A region will not provide selective replication in normal tissue.

In conclusion, we demonstrate a proof-of-principle that a tyrosinase CRAd could be useful in the gene therapy of glioma. Depending on the tyrosinase promoter location in the adenovirus genome, our data suggest that tyrosinasecontrolled transcription promotes viral replication and lyses glioma cells in vitro and in vivo. Overall, this finding provides the experimental evidence for the potential clinical use of recombinant vectors in glioma gene therapy.

\section{Acknowledgments}

This study was supported by a grant from the National Institutes of Neurological Disorders and Stroke (K08 NS046430, M.L.), the National Institutes of health (R01 CA108585, J.D.) and the Department of Defense (W81XWH-04-1-0800).

\section{References}

1. Maeda M, Namikawa K, Kobayashi I, et al: Targeted gene therapy toward astrocytoma using a Cre/loxP-based adenovirus system. Brain Res 1081: 34-43, 2006.

2. Kohno S, Nakagawa K, Hamada K, et al: Midkine promoterbased conditionally replicative adenovirus for malignant glioma therapy. Oncol Rep 12: 73-78, 2004

3. Van Houdt WJ, Haviv YS, Lu B, et al: The human survivin promoter: a novel transcriptional targeting strategy for treatment of glioma. J Neurosurg 104: 583-592, 2006.

4. De Leeuw B, Su M, ter Horst M, et al: Increased glia-specific transgene expression with glial fibrillary acidic protein promoters containing multiple enhancer elements. J Neurosci Res 83: 744-753, 2006.

5. Lazzari G, Colleoni S, Giannelli SG, et al: Direct derivation of neural rosettes from cloned bovine blastocysts: a model of early neurulation events and neural crest specification in vitro. Stem Cells 11: 2514-2521, 2006.

6. Chi DD, Merchant RE, Rand R, et al: Molecular detection of tumor-associated antigens shared by human cutaneous melanomas and gliomas. Am J Pathol 150: 2143-2152, 1997.

7. Ikemoto K, Nagatsu I, Ito S, King RA, Nishimura A and Nagatsu T: Does tyrosinase exist in neuromelanin-pigmented neurons in the human substantia nigra? Neurosci Lett 253: 198-200, 1998.

8. Prins RM, Odesa SK and Liau LM: Immunotherapeutic targeting of shared melanoma-associated antigens in a murine glioma model. Cancer Res 63: 8487-8491, 2003.

9. Miranda M, Botti D, Bonfigli A, Ventura T and Arcadi A: Tyrosinase-like activity in normal human substantia nigra. Gen Pharmacol 15: 541-544, 1984.

10. Tief K, Schmidt A, Aguzzi A and Beermann F: Tyrosinase is a new marker for cell populations in the mouse neural tube. Dev Dyn 205: 445-456, 1996.

11. Tief K, Schmidt A and Beermann F: Regulation of the tyrosinase promoter in transgenic mice: expression of a tyrosinase-lacZ fusion gene in embryonic and adult brain. Pigment Cell Res 10: 153-157, 1997. 
12. Nettelbeck DM, Rivera AA, Balague C, Alemany R and Curiel DT: Novel oncolytic adenoviruses targeted to melanoma: specific viral replication and cytolysis by expression of E1A mutants from the tyrosinase enhancer/promoter. Cancer Res 62: 4663-4670, 2002.

13. Nettelbeck DM, Jerome V and Muller R: A dual specificity promoter system combining cell cycle-regulated and tissuespecific transcriptional control. Gene Ther 6: 1276-1281, 1999.

14. Banerjee NS, Rivera AA, Wang M, et al: Analyses of melanomatargeted oncolytic adenoviruses with tyrosinase enhancer/ promoter-driven E1A, E4, or both in submerged cells and organotypic cultures. Mol Cancer Ther 3: 437-449, 2004.

15. Rivera AA, Davydova J, Schierer S, et al: Combining high selectivity of replication with fiber chimerism for effective adenoviral oncolysis of CAR-negative melanoma cells. Gene Ther 11: 1694-1702, 2004

16. Kanerva A, Zinn KR, Chaudhuri TR, et al: Enhanced therapeutic efficacy for ovarian cancer with a serotype 3 receptor-targeted oncolytic adenovirus. Mol Ther 8: 449-458, 2003

17. Tekant Y, Davydova J, Ramirez PJ, Curiel DT, Vickers SM and Yamamoto M: Oncolytic adenoviral therapy in gallbladder carcinoma. Surgery 137: 527-535, 2005.

18. Fueyo J, Gomez-Manzano C, Alemany R, et al: A mutant oncolytic adenovirus targeting the $\mathrm{Rb}$ pathway produces antiglioma effect in vivo. Oncogene 19: 2-12, 2000

19. LaBarre DD and Lowy RJ: Improvements in methods for calculating virus titer estimates from TCID50 and plaque assays. J Virol Methods 96: 107-126, 2001.

20. Kaname T, Kadomatsu K, Aridome K, et al: The expression of truncated MK in human tumors. Biochem Biophys Res Commun 219: 256-260, 1996.

21. Peng Y, Kang Q, Luo Q, et al: Inhibitor of DNA binding/ differentiation helix-loop-helix proteins mediate bone morphogenetic protein-induced osteoblast differentiation of mesenchymal stem cells. J Biol Chem 279: 32941-32949, 2004.

22. Kirby TO, Rivera A, Rein D, et al: A novel ex vivo model system for evaluation of conditionally replicative adenoviruses therapeutic efficacy and toxicity. Clin Cancer Res 10: 8697-8703, 2004.

23. El Andaloussi A, Sonabend AM, Han Y and Lesniak MS: Stimulation of TLR9 with CpG ODN enhances apoptosis of glioma and prolongs the survival of mice with experimental brain tumors. Glia 54: 526-535, 2006.

24. Mishima K, Asai A, Kadomatsu K, et al: Increased expression of midkine during the progression of human astrocytomas. Neurosci Lett 233: 29-32, 1997.

25. Kato S, Ishihara K, Shinozawa T, et al: Monoclonal antibody to human midkine reveals increased midkine expression in human brain tumors. J Neuropathol Exp Neurol 58: 430-441, 1999.

26. Sharma AM, Jagadisan B, Sheorain VS, Haldankar V and Mojamdar M: Transient expression of high molecular weight, heat sensitive, trypsin-resistant form of tyrosinase in B-16 melanoma cells. Pigment Cell Res 11: 375-379, 1998.

27. Prignano F, Gerlini G, Salvatori B, et al: Stem cell factor affects tumour progression markers in metastatic melanoma cells. Clin Exp Metastasis 23: 177-186, 2006.

28. Scott JN, Rewcastle NB, Brasher PM, et al: Which glioblastoma multiforme patient will become a long-term survivor? A population-based study. Ann Neurol 46: 183-188, 1999.

29. Ohgaki H, Dessen P, Jourde B, et al: Genetic pathways to glioblastoma: a population-based study. Cancer Res 64: 6892-6899, 2004.
30. Jayawardena S, Sooriabalan D, Indulkar S, Kim HH, Matin A and Maini A: Regression of grade III astrocytoma during the treatment of CML with imatinib mesylate. Am J Ther 13: 458-459, 2006.

31. Ho IA, Hui KM and Lam PY: Glioma-specific and cell cycleregulated herpes simplex virus type 1 amplicon viral vector. Hum Gene Ther 15: 495-508, 2004.

32. Todo T, Martuza RL, Rabkin SD and Johnson PA: Oncolytic herpes simplex virus vector with enhanced MHC class I presentation and tumor cell killing. Proc Natl Acad Sci USA 98: 6396-6401, 2001

33. Lamfers ML, Grill J, Dirven CM, et al: Potential of the conditionally replicative adenovirus Ad5-Delta24RGD in the treatment of malignant gliomas and its enhanced effect with radiotherapy. Cancer Res 62: 5736-5742, 2002.

34. Wang W, Tai CK, Kershaw AD, et al: Use of replicationcompetent retroviral vectors in an immunocompetent intracranial glioma model. Neurosurg Focus 20: E25, 2006.

35. Tai CK, Wang WJ, Chen TC and Kasahara N: Single-shot, multicycle suicide gene therapy by replication-competent retrovirus vectors achieves long-term survival benefit in experimental glioma. Mol Ther 12: 842-851, 2005.

36. Wang WJ, Tai CK, Kasahara N and Chen TC: Highly efficient and tumor-restricted gene transfer to malignant gliomas by replication-competent retroviral vectors. Hum Gene Ther 14 117-127, 2003

37. Yang WQ, Lun X, Palmer CA, et al: Efficacy and safety evaluation of human reovirus type 3 in immunocompetent animals: racine and non-human primates. Clin Cancer Res 10 8561-8576, 2004.

38. Wollmann G, Tattersall P and van den Pol AN: Targeting human glioblastoma cells: comparison of nine viruses with oncolytic potential. J Virol 79: 6005-6022, 2005.

39. Smit N, Le Poole I, van den Wijngaard R, Tigges A, Westerhof W and Das P: Expression of different immunological markers by cultured human melanocytes. Arch Dermatol Res 285: 356-365, 1993

40. Sarantou T, Chi DD, Garrison DA, et al: Melanoma-associated antigens as messenger RNA detection markers for melanoma. Cancer Res 57: 1371-1376, 1997.

41. Urosevic M, Braun B, Willers J, Burg G and Dummer R Expression of melanoma-associated antigens in melanoma cell cultures. Exp Dermatol 14: 491-497, 2005.

42. Liu G, Akasaki Y, Khong HT, et al: Cytotoxic T cell targeting of TRP-2 sensitizes human malignant glioma to chemotherapy Oncogene 24: 5226-5234, 2005.

43. Greggio E, Bergantino E, Carter D, et al: Tyrosinase exacerbates dopamine toxicity but is not genetically associated with Parkinson's disease. J Neurochem 93: 246-256, 2005.

44. De Marco F, Foppoli C, Coccia R, et al: Ectopic deposition of melanin pigments as detoxifying mechanism: a paradigm for basal nuclei pigmentation. Biochem Biophys Res Commun 314 631-637, 2004

45. Stoff-Khalili MA, Rivera AA, Le LP, et al: Employment of liver tissue slice analysis to assay hepatotoxicity linked to replicative and nonreplicative adenoviral agents. Cancer Gene Ther 13: 606-618, 2006

46. Fueyo J, Alemany R, Gomez-Manzano C, et al: Preclinical characterization of the antiglioma activity of a tropism-enhanced adenovirus targeted to the retinoblastoma pathway. J Natl Cancer Inst 95: 652-660, 2003. 